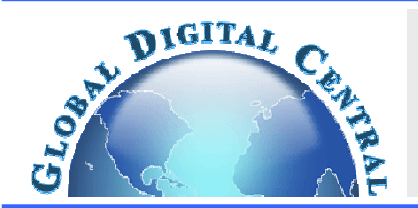

Frontiers in Heat and Mass Transfer

Available at www.ThermalFluidsCentral.org

\title{
UNSTEADY FLOW AND HEAT TRANSFER OF UCM FLUID IN A POROUS CHANNEL WITH VARIABLE THERMAL CONDUCTIVITY AND ION SLIP EFFECTS
}

\author{
Odelu Ojjela*, K. Pravin Kashyap, N. Naresh Kumar, and Samir Kumar Das \\ Department of Applied Mathematics \\ Defence Institute of Advanced Technology (Deemed University), Pune-411025, India
}

\begin{abstract}
This article presents an unsteady incompressible Upper Convected Maxwell (UCM) fluid flow with temperature dependent thermal conductivity between parallel porous plates which are maintained at different temperatures varying periodically with time. Assume that there is a periodic suction and injection at the upper and lower plates respectively. The governing partial differential equations are reduced to non linear ordinary differential equations by using similarity transformations and the solution is obtained using differential transform method. The effects of various fluid and geometric parameters on the velocity components, temperature distribution and skin friction are discussed in detail through graphs.
\end{abstract}

Keywords: Periodic suction and injection, Upper Convected Maxwell fluid, variable thermal conductivity, Hall and ion slip.

\section{INTRODUCTION}

The flow and heat transfer pertaining to non-Newtonian fluids through porous channels have immense importance both in industrial as well as biological flows, for example aerodynamics, lubrication, petroleum industries, polymers, electro-and magneto-responsive suspensions, cosmetic industry, the flow of blood in the arteries and the mechanics of cochlea in the human ear. In view of mathematical simplicity, the flow phenomenon between porous parallel plates has been pursued by many researchers to determine the constitutive properties of the fluid. Berman (1953) studied the two dimensional steady state incompressible laminar flow through a rectangular cross section with equally porous walls and noticed that the flow at the center line of the channel attains maximum. White et al. (1958) considered the viscous fluid flow in a uniform porous channel and obtained a solution for a wide range of suction Reynolds number. Terrill and Shrestha (1966) discussed the problem of steady incompressible two dimensional viscous fluid flow through a channel with uniformly porous walls and obtained a solution for large Reynolds number. Later the more general problem of symmetric and asymmetric suction driven flow through porous parallel plates is analyzed by Cox (1991). Ramana Murthy et al. (2007) examined the viscous fluid flow between porous parallel plates due to periodic suction and injection by retaining non linear convective terms and the analysis is carried out up to second order flow. Rassoulinejad-Mousavi and Abbasandy (2011) have considered a steady incompressible fully developed forced convection flow in a circular tube through a Darcy Brinkman Forchheimer porous medium and obtained an analytical solution by Spectral Homotopy Analysis method. Seyf and Rassoulinejad-Mousavi (2011) have analyzed a two dimensional laminar viscous fluid flow in a porous channel with moving or stationary walls subjected to injection/suction and the reduced flow field equations are solved by Homotopy Perturbation method (HPM). Asghar et al. (2011) have analyzed the fully developed viscous flow through a parallel plate channel under influence of Lorentz force and obtained analytical solution by using HPM. Rassoulinejad-Mousavi et. al. (2013) have examined a two dimensional steady laminar flow and heat transfer of an incompressible viscous fluid through a channel filled with homogenous porous medium and obtained a solution by HAM. Rassoulinejad-Mousavi and Yaghoobi (2014) have studied the influence of drag term on viscous dissipation for a channel filled with Darcy Brinkman Forchheimer porous medium with two different types of boundary conditions using DTM.

The viscoelastic models like Maxwell,Olyroyd, Boltzmann, PhanThien Tanner and other non linear models are studied by several researchers (Christensen (2012), Rajagopal and Srinivasa (2000), Anand and Rajagopal (2004), Hayat et. al. (2004), Hayat et. al. (2007)) due to their wide applications in engineering and science. Rassoulinejad-Mousavi et. Al. (2014) have investigated the effect of Lorentz force on a fully developed flow of conducting viscoelastic fluid between two parallel plates through a porous medium at various wall boundary conditions. One such non linear modification of Maxwell model is Upper Convected Maxwell model. Alizadeh and Sadeghy (2009) have found analytical solution for an unsteady MHD flow of Maxwellian fluid above stretching sheet by using Homotopy Analysis Method. Pahlavan et al. (2009) have considered the influence of thermal radiation, viscous dissipation on MHD flow of Maxwellian fluids above stretching sheets and studied the effects of various parameters like elastic number, Prandtl number etc. Sedeghy et al. (2005) studied the effects of Deborah's number on Sakadis flow of UCM fluid over a rigid plate and noticed that the elasticity of fluid destroys similarity between velocity profiles. A linear stability analysis of UCM fluid for a plane couette flow has been carried out by Renardy (1986) and no instabilities were observed. Hayat et al. (2006) have obtained a series solution for UCM fluid flow over a porous stretching sheet by Homotopy Analysis method. Hayat et al. (2007) have studied

* Corresponding author Email: odelu3@yahoo.co.in 
the Hall effects on peristaltic flow of an electrically conducting Maxwell fluid in a porous medium. Renardy (1997) has analyzed the high Weissenberg number effects of UCM fluids over elastic boundary layers of the channel. The influence of thermal radiation and Joule heating on MHD flow of Maxwell fluid in the presence of thermophoresis has been considered by Hayat and Qasim (2010). Unsteady pipe flows of Maxwell fluid has been investigated by Rahaman et al. (1995) for different types of pressure gradients. Unsteady flow of a Maxwell Fluid over a Stretching Surface in the Presence of a Heat Source/Sink has been investigated by Mukhopadhyay (2012) and obtained a numerical solution for the flow field equations by the shooting method. The unsteady laminar three dimensional flow of time dependent UCM fluid over a stretching sheet was analyzed by Awais et al. (2014) and obtained a series solution by HAM. Mushtaq et.al. (2016) have analyzed the Sakiadis flow of an UCM fluid over a semi infinite plate by considering Cattaneo Christov heat flux model and obtained a numerical solution by using shooting method with fifth order Runge Kutta method as well as Keller Box method.

The effects of variable viscosity and thermal conductivity on MHD micropolar fluid over a stretching sheet in porous channel have been studied by Patowary and Sut (2011). The influence of variable thermal conductivity and variable viscosity on the hydromagnetic flow and heat transfer over a stretching sheet has been analyzed by Prasad et al. (2010) and obtained a numerical solution for the governing coupled non linear differential equation by the Keller box method. The effect of Hall current on unsteady incompressible MHD flow and heat transfer between two parallel plates under the influence of temperature dependent viscosity and thermal conductivity is considered by Attia et al. (2003) and obtained a numerical solution by finite difference method. Recently, Shateyi et al. (2015) have studied the entropy generation on MHD Maxwell fluid flow and heat transfer over a stretching sheet in a Darcian porous medium and obtained a numerical solution for reduced non linear governing equations by using the Chebyshev spectral collocation method and the results are validated with Matlab BVp4c solver. The differential transform method (DTM) is first proposed by Zhou (1986) to solve linear and non linear initial value problems. Ravikanth and Aruna (2008) discussed the differential transform method in detail by several illustrations of both linear and non linear systems of partial differential equations and compared with exact solutions. Umavathi and Sekhar (2016) examined the free convection flow and heat transfer of a viscous fluid in a vertical channel with variable viscosity and thermal conductivity and the flow field equations are solved semi-analytically by differential transform method.

In this paper, we considered the effect of temperature dependent thermal conductivity on the MHD UCM fluid flow between parallel porous plates with Hall and ion slip currents, where the flow is induced by periodic suction and injection. The governing non linear partial differential equations are reduced to ordinary differential equations by similarity transformations and then obtained a semi numerical solution by Differential transform method. The effects of various fluid and geometric parameters on non dimensional velocity components and temperature distribution are discussed in detail and shown in the form of graphs. The results are compared with published work (Terrill and Shreshta (1966), Odelu and Naresh (2015)) for viscous fluid flow.

\section{FORMULATION OF THE PROBLEM}

Consider a fully developed unsteady laminar Upper Convected Maxwell fluid flow between parallel porous plates at $\mathrm{y}=0, \mathrm{y}=\mathrm{h}$ and $\mathrm{a}$ strong magnetic field is applied in $\mathrm{z}$ - direction as shown in the Fig 1. Assume that there is a periodic injection and suction at the lower and upper plates with velocities $v_{1} e^{i \omega t}$ and $v_{2} e^{i \omega t}$ respectively and the lower and upper plates are maintained with temperatures $T_{1} e^{i \omega t}$ and $T_{2} e^{i \omega t}$

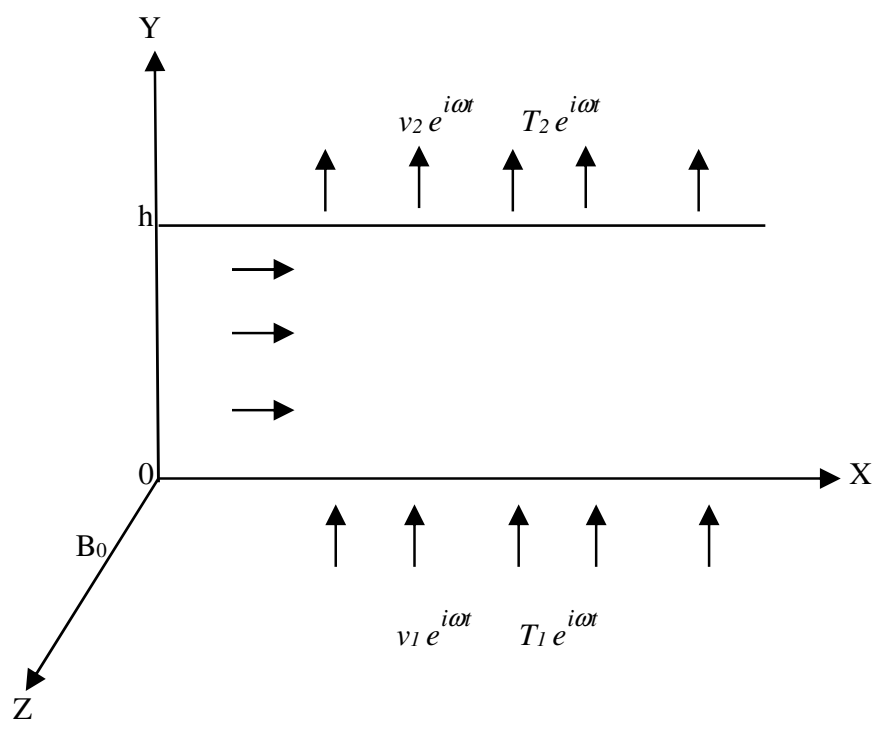

Fig. 1 Flow due to injection and suction at the plates

The governing equations of the unsteady incompressible upper convected Maxwell fluid flow with Hall and ion slip currents and in the absence of body forces are (Awais et al. (2014), Alizadeh and Sadeghy (2009))

$$
\begin{aligned}
& \frac{\partial u}{\partial x}+\frac{\partial v}{\partial y}=0 \\
& \rho\left[\frac{\partial u}{\partial t}+u \frac{\partial u}{\partial x}+v \frac{\partial u}{\partial y}+\beta\left(u^{2} \frac{\partial^{2} u}{\partial x^{2}}+v^{2} \frac{\partial^{2} u}{\partial y^{2}}+2 u v \frac{\partial^{2} u}{\partial x \partial y}\right)\right]= \\
& -\frac{\partial P}{\partial x}+\mu\left(\frac{\partial^{2} u}{\partial x^{2}}+\frac{\partial^{2} u}{\partial y^{2}}\right)-\sigma B_{0}^{2}\left(\frac{(1+B i B e) u-B e v}{(1+B i B e)^{2}+B e^{2}}\right) \\
& \rho\left[\frac{\partial v}{\partial t}+u \frac{\partial v}{\partial x}+v \frac{\partial v}{\partial y}+\beta\left(u^{2} \frac{\partial^{2} v}{\partial x^{2}}+v^{2} \frac{\partial^{2} v}{\partial y^{2}}+2 u v \frac{\partial^{2} v}{\partial x \partial y}\right)\right]= \\
& -\frac{\partial P}{\partial y}+\mu\left(\frac{\partial^{2} v}{\partial x^{2}}+\frac{\partial^{2} v}{\partial y^{2}}\right)-\sigma B_{0}^{2}\left(\frac{(1+B i B e) v+B e u}{(1+B i B e)^{2}+B e^{2}}\right) \\
& \rho c_{p}\left[\frac{\partial T}{\partial t}+u \frac{\partial T}{\partial x}+v \frac{\partial T}{\partial y}\right]=\left(\frac{\partial k}{\partial x} \frac{\partial T}{\partial x}+\frac{\partial k}{\partial y} \frac{\partial T}{\partial y}\right)+k\left(\frac{\partial^{2} T}{\partial x^{2}}+\frac{\partial^{2} T}{\partial x^{2}}\right)+ \\
& \left(\frac{\sigma B_{0}^{2}\left(u^{2}+v^{2}\right)}{(1+B i B e)^{2}+B e^{2}}\right)
\end{aligned}
$$

Where $\beta$ is the relaxation coefficient of Maxwell fluid, $\mathrm{P}$ is pressure, $\rho$ is density, $\mathrm{k}$ is thermal conductivity, $\mathrm{c}$ is specific heat at constant temperature. The induced magnetic fields are neglected as they are very small compared to the applied magnetic field, so as to see that the Reynolds number is small, electric field is zero and magnetic permeability is the same throughout the flow field.

The following similarity transformations are used to reduce the partial differential equations into ordinary differential equations (Patowary and Sut (2011), Terrill and Shrestha (1968))

$$
\begin{aligned}
& u(x, \lambda, t)=\left(\frac{u_{0}}{a}-\frac{v_{2} x}{h}\right) f^{\prime}(\lambda) e^{i \omega t} \\
& v(x, \lambda, t)=v_{2} f(\lambda) e^{i \omega t} \\
& k=k_{0}\left(1+\alpha\left(\frac{\left(T-T_{1} e^{i \omega t}\right)}{\left(T_{2}-T_{1}\right) e^{i \omega t}}\right)\right)
\end{aligned}
$$




$$
T(x, \lambda, t)=\left[T_{1}+\frac{\mu v_{2}}{\rho h c}\left(\phi_{1}(\lambda)+\left(\frac{u_{0}}{a v_{2}}-\frac{x}{h}\right)^{2} \phi_{2}(\lambda)\right] e^{i \omega t}\right.
$$

Where $\lambda=\frac{y}{h}$ and $f(\lambda), \phi_{1}(\lambda), \phi_{2}(\lambda)$ are unknown functions which have to be determined. The boundary conditions on the velocity components and temperature are

$$
\begin{aligned}
& u(x, 0, t)=0, v(x, 0, t)=v_{1} e^{i \omega t}, T(x, 0, t)=T_{1} e^{i \omega t} \\
& u(x, 1, t)=0, v(x, 1, t)=v_{2} e^{i \omega t}, T(x, 1, t)=T_{2} e^{i \omega t}
\end{aligned}
$$

Substituting (5) in (2), (3) and (4), we get

$$
\begin{aligned}
& f^{i v}=\frac{\operatorname{Re} \cos \phi\left(f^{\prime} f^{\prime \prime}-f f^{\prime \prime \prime}\right)}{\left(1-W i \operatorname{Re} f^{2} \cos 2 \phi\right)}-\frac{2 W i \operatorname{Re} \cos 2 \phi}{\left(1-W i \operatorname{Re} f^{2} \cos 2 \phi\right)} \\
& \left(f^{\prime^{2}} f^{\prime \prime}+f f^{\prime \prime^{2}}\right)+\frac{H a^{2}(1+B i B e)}{\left((1+B i B e)^{2}+B e^{2}\right)} \\
& \left(\frac{f^{\prime \prime}}{1-W i \operatorname{Re} f^{2} \cos 2 \phi}\right) \\
& \phi_{1}^{\prime \prime}=\frac{1}{\left(1+\alpha E c \phi_{1}\right)}\left[\operatorname{Re} \operatorname{Pr} f \phi_{1}^{\prime} \cos \phi-\alpha E c\left(\phi_{1}^{\prime}\right)^{2}\right] \\
& -\left(\frac{H a^{2} \operatorname{Re} \operatorname{Pr} f^{2} \cos \phi}{\left(1+\alpha E c \phi_{1}\right)\left((1+B i B e)^{2}+B e^{2}\right)}\right)-2 \phi_{2} \\
& \phi_{2}^{\prime \prime}=\frac{1}{\left(\phi_{1}+\alpha E c+\alpha E c \xi^{2} \phi_{2}\right)}\left[\operatorname{Re} \operatorname{Pr}\left[\phi_{2}^{\prime} f-2 f^{\prime} \phi_{2}\right] \cos \phi-\alpha E c\right. \\
& {\left[4\left(\phi_{2}^{\prime}\right)^{2}+2 \phi_{2}^{\prime} \phi_{1}^{\prime}+\left(\xi \phi_{2}^{\prime}\right)^{2}\right]-\left(\frac{H a^{2} \operatorname{Re} \operatorname{Pr} \cos \phi}{(1+B i B e)^{2}+B e^{2}}\right)\left(f^{\prime}\right)^{2}} \\
& \left.-\alpha E c\left(\phi_{2}\left(2 \phi_{2}+\phi_{1}^{\prime \prime}\right)\right)\right]
\end{aligned}
$$

The prime in the above equations denotes the differentiation with respect to $\lambda$.

The boundary conditions (7) in terms of are $f, \phi_{1}$ and $\phi_{2}$ are

$$
\begin{array}{ll}
f(0)=1-a, & f(1)=1, \\
f^{\prime}(0)=0, & f^{\prime}(1)=0, \\
\varphi_{1}(0)=0, & \phi_{1}(1)=1 / E c, \\
\varphi_{2}(0)=0, & \varphi_{2}(1)=0
\end{array}
$$

\section{SOLUTION OF THE PROBLEM}

Each term in the nonlinear equations (7), (8) and (9) is transformed and the recurring relations involving the Taylor series coefficients $F[k], G[k]$ and $\mathrm{H}[\mathrm{k}]$ are calculated and are substituted back into the series.

$$
\begin{array}{lrl}
F[k]=\frac{1}{k !} \frac{d^{k} f}{d x^{k}} & f(x)=\sum_{j=0}^{k} F[j] x^{j} \\
G[k]=\frac{1}{k !} \frac{d^{k} \phi_{1}}{d x^{k}} & \phi_{1}(x)=\sum_{j=0}^{k} G[j] x^{j} \\
H[k]=\frac{1}{k !} \frac{d^{k} \phi_{2}}{d x^{k}} & \phi_{2}(x)=\sum_{j=0}^{k} H[j] x^{j}
\end{array}
$$

and the related transformations are

$$
\begin{aligned}
& f \rightarrow F[k], \quad \phi_{1} \rightarrow G[k], \quad \phi_{2} \rightarrow H[k], \\
& f \phi_{1} \rightarrow \sum_{l=0}^{k} F[l] G[k-l], \\
& f \phi_{1} \phi_{2} \rightarrow \sum_{s=0}^{k} \sum_{m=0}^{k-s} F[s] G[m] H[k-s-m], \\
& \frac{d^{n} f}{d x^{n}} \rightarrow \frac{(k+1)}{k !} F[k+n]
\end{aligned}
$$

The transformed equations of (7), (8) and (9) respectively are as follows

$$
\begin{array}{r}
F[k+4]=\left(\operatorname{Re} \cos \phi \sum_{l=0}^{k}((l+3)(l+2)(l+1) F[l+3] F[k-l]-(l+2)\right. \\
(l+1) F[l+2](k-l+1) F[k-l+1])+\operatorname{Re} W i \cos 2 \phi \\
\sum_{s=0}^{k} \sum_{m=0}^{k-s}[(s+4)(s+3)(s+2)(s+1) F[m] F[s+4]- \\
2 \operatorname{Re} W i \cos 2 \phi \sum_{s=0}^{k} \sum_{m=0}^{k-s}((m+1)(s+1) F[m+1] F[s+1] \\
F[k-m-s]+(m+1)(m+2) F[k-m-s]-(s+2) \\
(s+1) F[m+2] F[s+2] F[k-s-m])]+H a^{2}(k+2) \\
\left.(k+1) \frac{(1+B i B e)}{(1+B i B e)^{2}+B e^{2}} F[k+2]\right) \\
/\left(1-F[0]^{2} \operatorname{Re} W i \cos 2 \phi\right)(k+4)(k+3)(k+2)(k+1)
\end{array}
$$

$$
\begin{aligned}
G[k+2]= & \sum_{l=0}^{k}(\operatorname{Re} \operatorname{Pr} \cos \phi(l+1) G[l+1] F[k-l]-\operatorname{Re} \operatorname{Pr} \cos \phi \\
& \frac{H a^{2}}{(1+B i B e)^{2}+B e^{2}} F[l] F[k-l]-2 \alpha E c G[l] H[k-l] \\
& -\alpha E c G[l] G[k-l])-\alpha E c \sum_{l=0}^{k-1}(l+2)(l+1) G[l+2] \\
& G[k-l]-2 H[k]) /(k+2)(k+1)(1+\alpha E c G[0])
\end{aligned}
$$$$
H[k+2]=\left(\operatorname{Re} \operatorname{Pr} \cos \phi \sum_{l=0}^{k}(l+1)(H[l+1] F[k-l] H[l+1]\right.
$$$$
F[k-l])-2 F[l+1] H[k-l]-\alpha E c \sum_{l=0}^{k}(4 H[l] H[k-l]
$$$$
-2(l+1)(k-l+1) G[l+1] H[k-l+1]+\xi^{2}(l+1)
$$ 


$$
\begin{aligned}
& (k-l+1) H[l+1] H[k-l+1])+\frac{H a^{2} \operatorname{Re} \operatorname{Pr} \cos \phi}{(1+B i B e)^{2}+B e^{2}} \\
& \sum_{l=0}^{k}(l+1)(k-l+1) F[l+1] F[k-l+1]-\alpha E c \operatorname{Re} \operatorname{Pr} \\
& \cos \phi \sum_{s=0}^{k} \sum_{m=0}^{k-s}((s+1) H[s+1] G[m] F[k-s-m] \\
& -2(s+1) F[s+1] G[m] H[k-s-m]))-\alpha^{2} E c^{2} \\
& k k-s \\
& \left(\sum_{s=0}^{k} \sum_{m=0}^{k-s} 4 H[s] H[m] G[k-s-m]+2(s+1)(m+1)\right. \\
& G[s+1] H[m+1] G[k-s-m]+\xi^{2}(s+1)(m+1) H[s+1] \\
& H[m+1] G[k-s-m]))+\frac{\alpha E c H a^{2} \operatorname{Re} \operatorname{Pr} \cos \phi}{(1+B i B e)^{2}+B e^{2}} \\
& H[k-s-m]\left(\sum_{s=0}^{k} \sum_{m}^{k-s}(s+1)(m+1) F[s+1] F[m+1]\right. \\
& G[k-s-m])-\alpha E c\left(\sum_{s=0}^{k} \sum_{m=0}^{k-s}(\operatorname{Re} \operatorname{Pr} \cos \phi(s+1)\right. \\
& G[s+1] F[m]-\alpha E c G[s] G[m] H[k-s-m] \\
& -\frac{H a^{2} \operatorname{Re} \operatorname{Pr} \cos \phi}{(1+B i B e)^{2}+B e^{2}} F[s] F[m] H[k-s-m] \\
& -\left(\sum _ { l = 0 } ^ { k - 1 } ( l + 2 ) ( l + 1 ) \left(H[l+2] G[k-l]+\alpha E c \xi^{2} H[l+2]\right.\right. \\
& \left.\left.H[k-l]+\alpha^{2} E c^{2} H[l+2] G[k-l]\right)\right)-\left(\sum_{s=0}^{k} \sum_{m=0}^{k-s}\right. \\
& (s+2)(s+1)\left(\alpha^{2} E c^{2} \xi^{2} H[s+2] H[m] G[k-s-m]\right. \\
& +\alpha E c H[s+2] G[m] G[k-s-m]))) /\left(( s + 2 ) ( s + 1 ) \left(G[0]^{2}\right.\right. \\
& \left.\left.+\alpha E c+\alpha E c \xi^{2} H[0]+\alpha^{2} E c^{2} \xi^{2} H[0] G[0]+\alpha^{2} \xi^{2} G[0]\right)\right)
\end{aligned}
$$

with the initial conditions

$$
\begin{aligned}
& F[0]=1-a, F[1]=0, G[0]=0, H[0]=0, \\
& F[2]=n_{1}, \quad F[3]=n_{2}, \quad G[1]=n_{3}, \quad H[1]=n_{4}
\end{aligned}
$$

The constants $n_{1}, n_{2}, n_{3}, n_{4}$ are calculated from the equations (13) to (16) and substituted in (11) such that they satisfy the boundary condition at $\lambda=1$ then we obtained the velocity components and temperature distribution for various parameters.

\section{RESULTS AND DISCUSSION}

The non-dimensional axial velocity, radial velocity and temperature distribution have been computed using the differential transform method for various fluid and geometric parameters such as Weissenberg number Wi , Hall parameter Be, Ion slip parameter Bi, Prandtl number $\operatorname{Pr}$, thermal conductivity parameter $\alpha$, suction injection parameter a, frequency parameter $\phi$ and the results are shown in the form of graphs in the domain $[0,1]$.

Figure 2 shows the influence of $\mathrm{Wi}$ on axial and radial velocities. It is observed that with increasing Wi the axial velocity increases till the mid-point and then decreases towards the upper plate whereas the radial velocity decreases towards the upper plate. It is a known fact that the viscoelastic effect dominates the inertial effect for low Reynolds number flows. The strong magnetic field in Z- direction results in Hall and ion slip effects. The effect of Be on velocity components and temperature distribution are illustrated in the Figure 3. It is observed that the axial velocity decreases up to the center of the channel then increases towards the upper plate while the radial velocity and temperature decrease towards the upper plate with the increasing of Be. A similar pattern of axial velocity, radial velocity and temperature is observed for ion slip parameter as depicted in Figure 4. This is because of the increase in the Hall and ion slip currents reduce the effect of Lorentz force (Sutton and Sherman (1965)). Figures 5 and 6 display the effects of $\operatorname{Pr}$ and $\alpha$ on temperature distribution respectively. From the figures it is analyzed that the temperature distribution decreases towards the upper plate with the increasing of $\operatorname{Pr}$ and $\alpha$. This is due to the fact that the thermal boundary layer thickness decreases as Pr increases.

The effect of a on velocity components and temperature distribution is presented in the Figure 7. From these, it is noticed that as ' $a$ ' increases, the axial velocity and temperature distribution increase whereas the radial velocity decreases towards the upper plate. The Figure 8 shows the effect of $\phi$ on velocity components and temperature distribution and from these it is analyzed that when $\phi$ increases the radial velocity also increases whereas the temperature distribution decreases towards the upper plate. However the axial velocity increases in the first half and then decreases towards upper plate.

Table 1 shows the comparative results for non dimensional skin friction for viscous fluid with the existing literature (Terrill and Shreshta, 1968; Odelu and Naresh 2015) and is found to be in good agreement. The validation of the code has been performed by computing the velocity components using differential transform method (DTM) and bvp4c in MATLAB for the present problem is shown in the Table 2. It is noticed that the results with DTM are very close to the bvp4c in MATLAB.

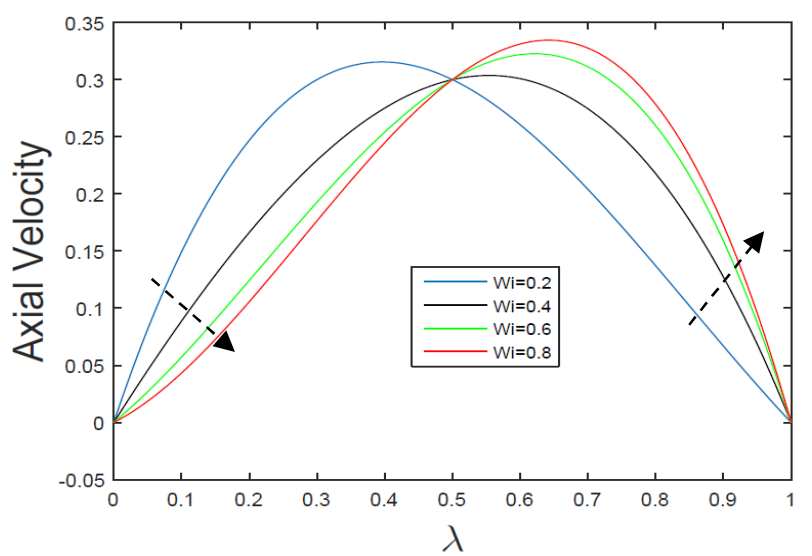

(a)

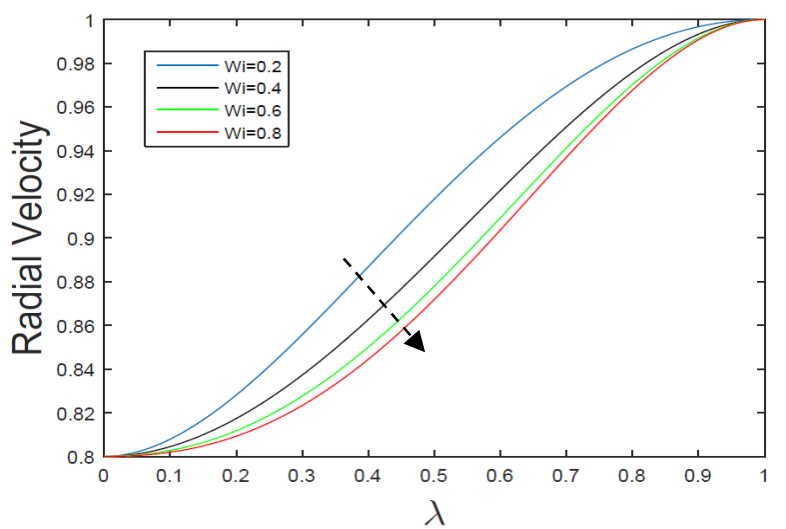

(b)

Fig. 2 Effect of Wi on (a) Axial velocity, (b) Radial velocity fora=0.2; $\alpha=0.8 ; \mathrm{Re}=2 ; \mathrm{Ha}=4 ; \mathrm{Bi}=2 ; \mathrm{Be}=2 ; \phi=0.2 ; \mathrm{Ec}=1 ; \xi=0.6325 ; \mathrm{Pr}=4$. 


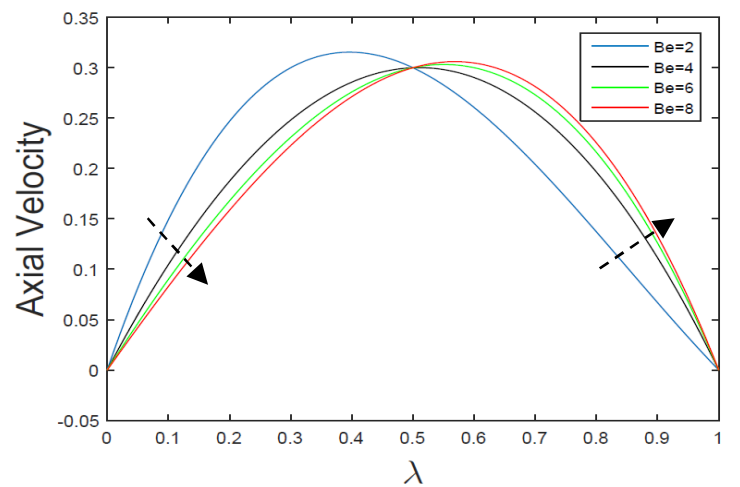

(a)

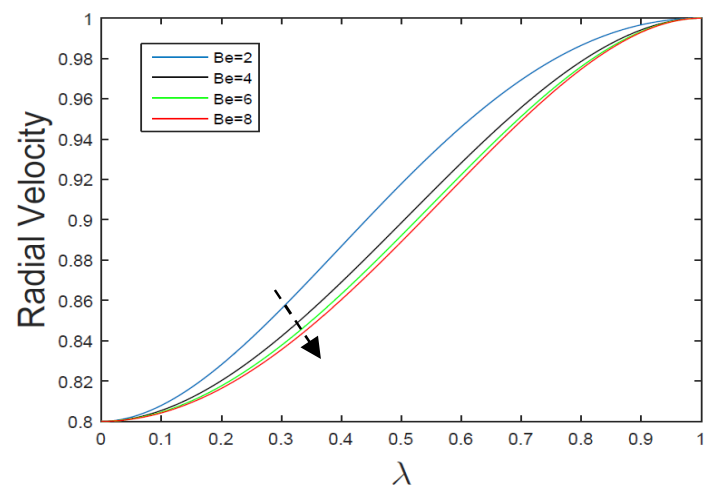

(b)

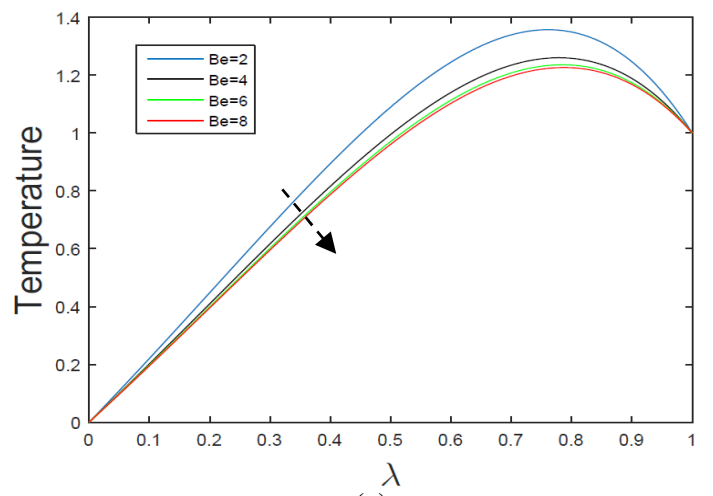

(c)

Fig. 3 Effect of Be on (a) Axial velocity, (b) Radial velocity, (c) Temperature for a=0.2; $\alpha=0.2 ; \mathrm{Re}=2 ; \mathrm{Ha}=4 ; \mathrm{Bi}=2 ; \mathrm{Wi}=0.2 ; \phi=0.2 ; \mathrm{Ec}=1 ; \xi=0.6325 ; \mathrm{Pr}=0.2$

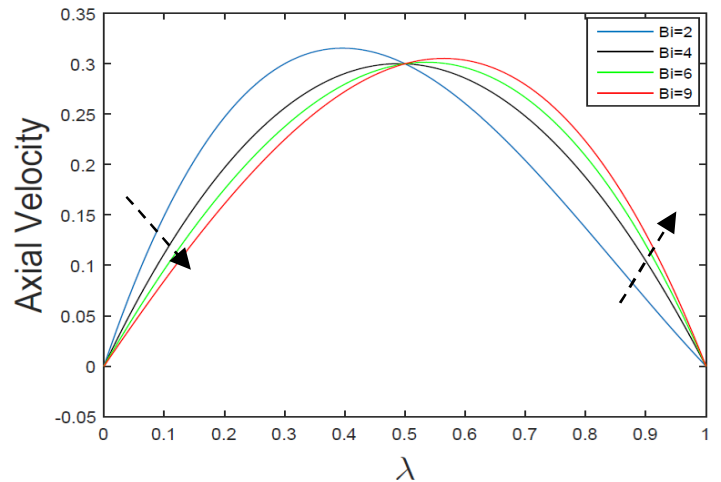

(a)

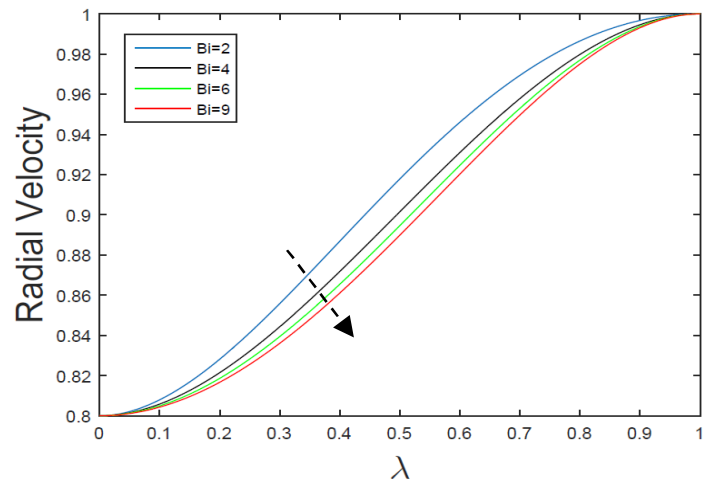

(b)

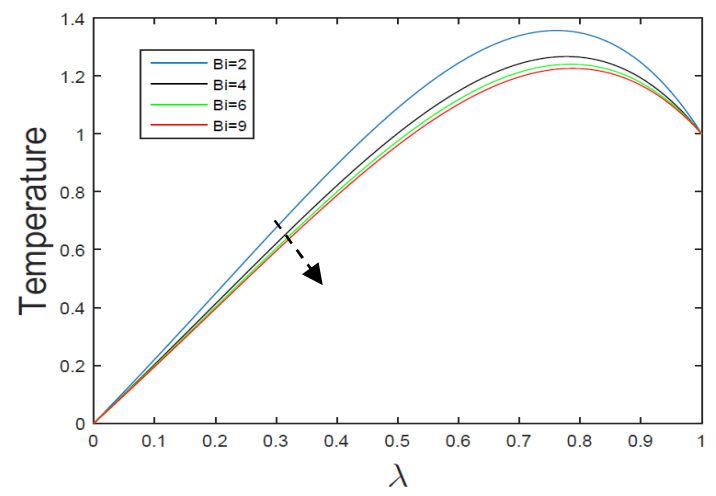

(c)

Fig. 4 Effect of Bi on (a) Axial velocity, (b) Radial velocity, (c) Temperature for $a=0.2 ; \alpha=0.2 ; \mathrm{Re}=2 ; \mathrm{Ha}=4 ; \mathrm{Wi}=0.2 ; \mathrm{Be}=2 ; \phi=0.2 ; \mathrm{Ec}=1$; $\xi=0.6325 ; \operatorname{Pr}=0.2$. 


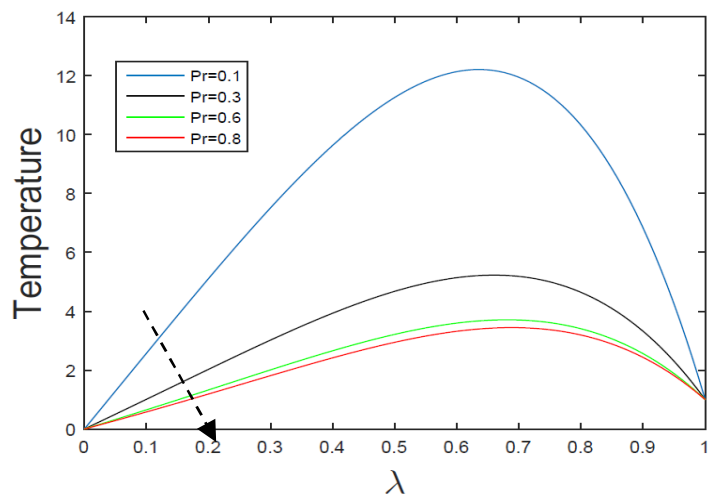

Fig. 5 Effect of $\operatorname{Pr}$ on Temperature $\$ \mathrm{a}=0.2 ; \alpha=0.4 ; \mathrm{Re}=1 ; \mathrm{Ha}=4 ; \mathrm{Bi}=2 ; \mathrm{Be}=2 ; \mathrm{Wi}=0.2 ; \phi=0.2 ; \mathrm{Ec}=1 ; \xi=0.6325 ; \mathrm{Pr}=0.2$.

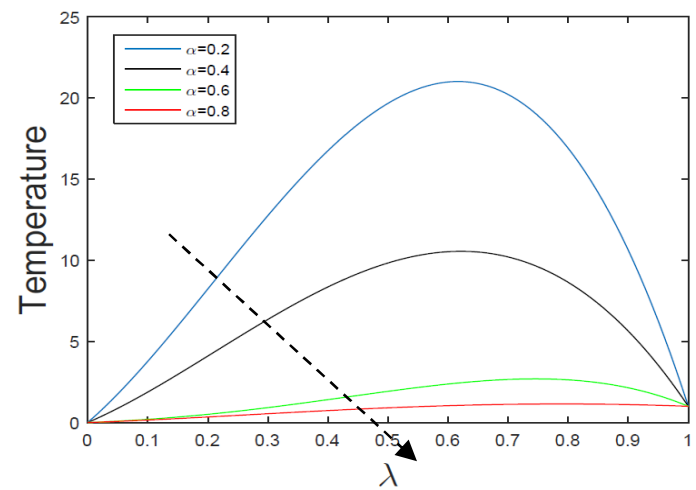

Fig. 6 Effect of $\alpha$ on Temperature a=0.2; $\mathrm{Re}=2 ; \mathrm{Ha}=4 ; \mathrm{Bi}=2 ; \mathrm{Be}=2 ; \mathrm{Wi}=0.2 ; \phi=0.2 ; \mathrm{Ec}=1 ; \quad \xi=0.6325 ; \mathrm{Pr}=0.2$.

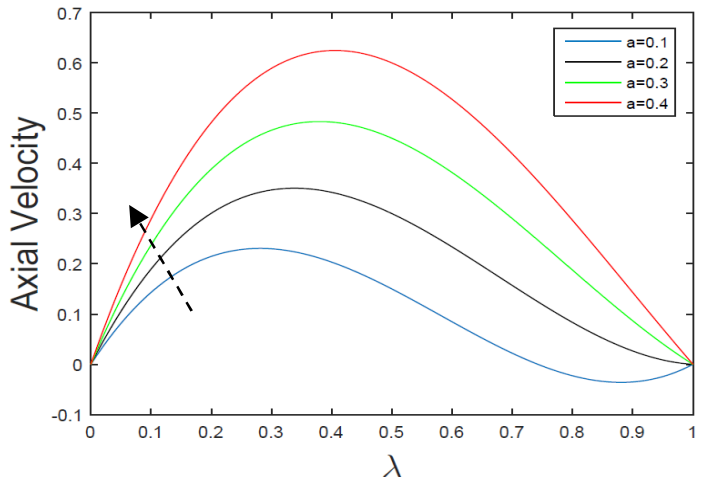

(a)

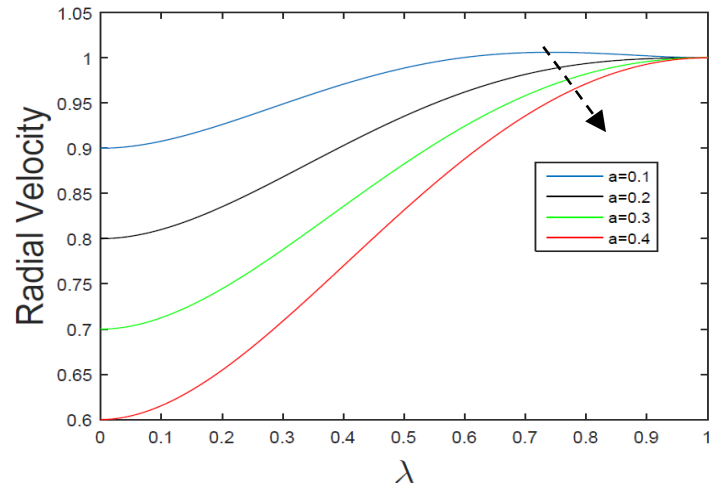

(b)

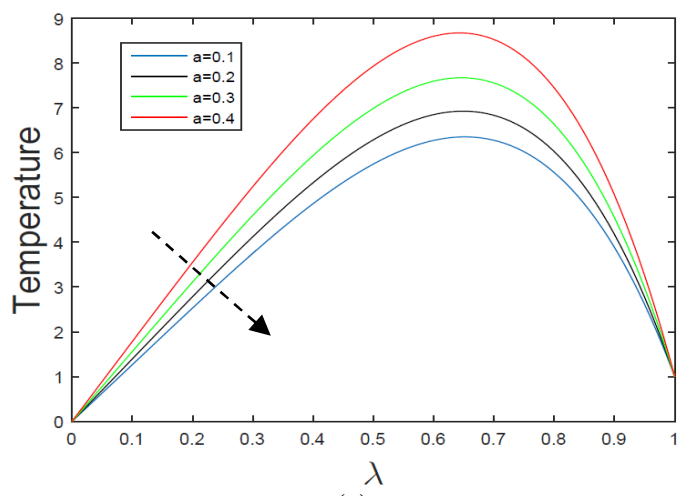

(c)

Fig. 7 Effect of suction-injection ratio on (a) Axial velocity, (b) Radial velocity, (c) Temperature $\alpha=0.4 ; \mathrm{Re}=1 ; \mathrm{Ha}=4 ; \mathrm{Bi}=2$; $\mathrm{Bi}=2$; Wi=0.2; $\phi=0.2$; $\mathrm{Ec}=1 ; \xi=0.6325 ; ; \operatorname{Pr}=0.2$. 


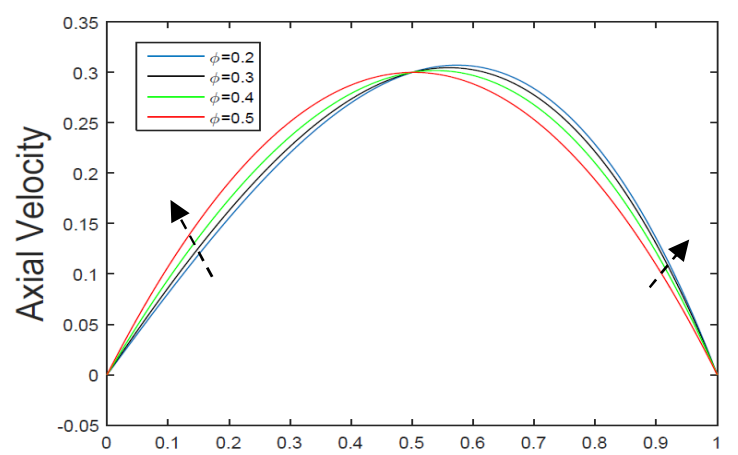

(a)

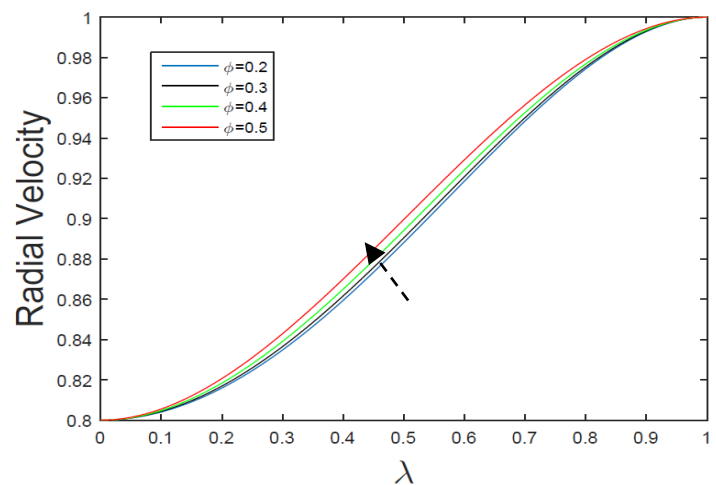

(b)

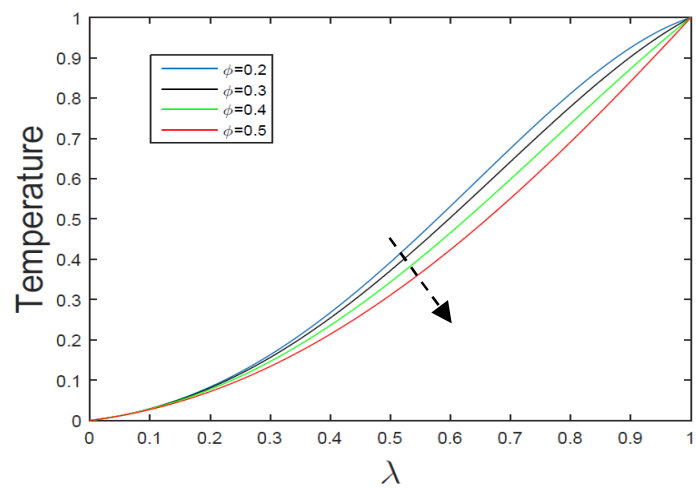

(c)

Fig. 8 Effect of $\phi$ on (a) Axial velocity, (b) Radial velocity, (c) Temperature a=0.2; $\alpha=0.2 ; \mathrm{Re}=2 ; \mathrm{Ha}=2 ; \mathrm{Bi}=2 ; \mathrm{Be}=2 ; \mathrm{Wi}=0.2 ; \mathrm{Ec}=1 ; \xi=0.6325$; $\mathrm{Pr}=5$.

Table 1 Comparision of skin friction values at the lower boundary for Newtonian case.

\begin{tabular}{|c|c|c|c|c|}
\hline \multirow{2}{*}{ Re } & \multirow{2}{*}{ a } & \multicolumn{3}{|c|}{ Skin friction } \\
\cline { 2 - 5 } & & $\begin{array}{c}\text { Terrill and Shrestha } \\
(1968)\end{array}$ & $\begin{array}{c}\text { Odelu and Naresh } \\
(2015)\end{array}$ & Present \\
\hline-16.16 & 1.9766 & 10.726 & 10.6621 & 10.7085 \\
\hline-27.15 & 1.8358 & 10.486 & 10.464 & 10.4538 \\
\hline-48.54 & 1.9461 & 10.202 & 10.1985 & 10.2372 \\
\hline-55.34 & 1.7764 & 10.329 & 10.3246 & 10.37380 \\
\hline-74.72 & 1.9308 & 10.100 & 10.1989 & 10.1120 \\
\hline-149.13 & 1.9278 & 9.995 & 9.9952 & 9.97306 \\
\hline-156.44 & 1.8602 & 10.036 & 10.0361 & 10.1590 \\
\hline-206.42 & 1.6194 & 10.548 & 10.5485 & 10.77050 \\
\hline
\end{tabular}

Table 2 Comparision of bvp4c (numerical) and DTM (present) for velocity components at $\mathrm{a}=0.2 ; \alpha=0.2 ; \mathrm{Re}=2 ; \mathrm{Ha}=2 ; \mathrm{Bi}=2 ; \mathrm{Be}=2 ; \mathrm{Wi}=0.2 ; \mathrm{Ec}=1 ; \xi=0.6325 ; \mathrm{Pr}=5$.

\begin{tabular}{|c|c|c|c|c|c|c|}
\hline \multirow{2}{*}{$\Lambda$} & \multicolumn{3}{|c|}{ Axial velocity } & \multicolumn{3}{|c|}{ Radial velocity } \\
\cline { 2 - 7 } & bvp4c & DTM & Error & bvp4c & DTM & Error \\
\hline 0 & 0 & 0 & 0 & 0.8 & 0.8 & 0 \\
\hline 0.1 & 0.067650 & 0.065925 & 0.001724 & 0.803419 & 0.803317 & 0.000101 \\
\hline 0.2 & 0.1306704 & 0.128813 & 0.001857 & 0.813376 & 0.813084 & 0.000291 \\
\hline 0.3 & 0.188170 & 0.187359 & 0.000811 & 0.829369 & 0.828936 & 0.000433 \\
\hline 0.4 & 0.238477 & 0.239362 & 0.000884 & 0.850772 & 0.850339 & 0.000432 \\
\hline 0.5 & 0.278723 & 0.281276 & 0.002553 & 0.876732 & 0.876474 & 0.000257 \\
\hline 0.6 & 0.304157 & 0.307534 & 0.003376 & 0.906026 & 0.906071 & 0.000044 \\
\hline 0.7 & 0.306981 & 0.3095103 & 0.002528 & 0.936815 & 0.937146 & 0.000331 \\
\hline 0.8 & 0.274279 & 0.2739807 & 0.000298 & 0.966247 & 0.966590 & 0.000342 \\
\hline 0.9 & 0.184349 & 0.1808658 & 0.003483 & 0.98978 & 0.989490 & 0.000290 \\
\hline 1 & 0 & 0 & 0 & 1 & 0.998008 & 0.001991 \\
\hline
\end{tabular}




\section{CONCLUSIONS}

In this paper, the unsteady incompressible Upper Convected Maxwell fluid flow and heat transfer between porous parallel plates in the presence of strong magnetic field is considered. The flow field equations are reduced to nonlinear ordinary differential equations using similarity transformations then solved by the differential transform method. The effects on velocity components and temperature distribution with respect to various parameters has been studied and shown graphically and we observed that

- The temperature of the fluid is enhanced with ' $a$ ' whereas it is decreased with increasing $\mathrm{Pr}$.

- The velocity components and temperature distribution are exhibiting similar effects for Hall and ion slip parameters.

- As thermal conductivity increases, the temperature of the fluid is decreased.

- The radial velocity decreases by increasing the Wi and the axial velocity also decreases up to the center of the plates then the flow is reversed.

- The present results have better agreement with existing literature Terrill and Shrestha (1966) and Odelu and Naresh (2015) for the Newtonian case.

\section{ACKNOWLEDGEMENTS}

One of the authors (KPK) is grateful to University Grants Commission, Government of India for providing senior research fellowship (F.218/2012(SA-I)).

\section{NOMENCLATURE}

$h$
Distance between parallel plates Injection velocity at lower plate Suction velocity at the upper plate $1-\frac{v_{1}}{v_{2}}$

Fluid pressure

Axial velocity component

Velocity component in $y$ - direction

Weissenberg's number, $\frac{\beta v_{2}}{h}$

Dimensionless temperature, $\frac{T-T_{1} e^{i \omega t}}{\left(T_{2}-T_{1}\right) e^{i \omega t}}$

Temperature at the lower plate

Temperature at the upper plate

Thermal conductivity

Initial thermal conductivity

Eckert number, $\frac{\mu v_{2}}{\rho h c\left(T_{2}-T_{1}\right)}$

Prandtl number, $\frac{\mu c_{p}}{k}$

Magnetic Field strength

Hartmann number, $B_{0} h \sqrt{\frac{\sigma}{\mu}}$

Hall parameter

ion slip parameter

Suction Reynolds number, $\frac{\rho h v_{2}}{\mu}$

Entrance velocity

$\begin{array}{ll}\alpha & \text { Thermal conductivity parameter } \\ \beta & \text { Maxwell parameter } \\ \phi & \text { Frequency parameter } \\ \lambda & \text { Dimensionless y coordinate, } y / h \\ \xi & \text { Dimensionless axial variable, }\left(\frac{u_{0}}{a v_{2}}-\frac{x}{h}\right) \\ \rho & \text { Fluid density } \\ \mu & \text { Fluid viscosity }\end{array}$

\section{REFERENCES}

Alizadeh-Pahlavan, A., and Sadeghy, K., 2009, "On the use of Homotopy Analysis Method for Solving Unsteady MHD Flow of Maxwellian Fluids above Impulsively Stretching Sheets," Communications in Nonlinear Science and Numerical Simulation, 14(4), 1355-1365.

http://dx.doi.org/10.1016/j.cnsns.2008.03.001

Aliakbar, V., Alizadeh-Pahlavan, A., and Sadeghy, K., 2009, "The Influence of Thermal Radiation on MHD Flow of Maxwellian Fluids above Stretching Sheets," Communications in Nonlinear Science and Numerical Simulation, 14(3), 779-794.

http://dx.doi.org/10.1016/j.cnsns.2007.12.003

Anand, M., and Rajagopal, K., 2004, “A Shear-thinning Viscoelastic Fluid Model for Describing the Flow of Blood," International Journal of Cardiovascular Medicine and Science, 4(2), 59-68.

Asghar Shirazpour, Seyed Moein Rassoulinejad-Mousavi, Hamid Reza Seyf,(2011), "HPM Solution of Momentum Equation for DarcyBrinkman Model in a Parallel Plates Channel Subjected to Lorentz Force," International Journal of Mechanical, Aerospace, Industrial, Mechatronic and Manufacturing Engineering 5(1), 258-262.

Attia, H. A., and Aboul-Hassan, A. L., 2003, "The Effect of Variable Pproperties on the Unsteady Hartmann Flow with Heat Transfer considering the Hall Effect," Applied Mathematical Modelling, 27(7), $551-563$.

http://dx.doi.org/10.1016/S0307-904X(03)00090-8

Awais, M., Hayat, T., Alsaedi, A., and Asghar, S., 2014. "TimeDependent Three-dimensional Boundary Layer Flow of a Maxwell Fluid," Computers and Fluids, 91, 21-27. http://dx.doi:10.1016/j.compfluid.2013.12.002

Berman, A. S., 1953, "Laminar Flow in Channels with Porous Walls," Journal of Applied physics, 24(9), 1232-1235. http://dx.doi.org/10.1063/1.1721476

Cox, S. M., 1991, "Two-dimensional Flow of a Viscous Fluid in a Channel with Porous Palls," Journal of Fluid Mechanics, 227, 1-33. http://dx.doi.org/10.1017/S0022112091000010

Christensen, R., 2012. Theory of Viscoelasticity: an Introduction. Elsevier.

Hayat, T., Nadeem, S., and Asghar, S., 2004, "Periodic Unidirectional Flows of a Viscoelastic Fluid with the Fractional Maxwell Model," Applied Mathematics and Computation, 151(1), 153-161. http://dx.doi.org/10.1016/S0096-3003(03)00329-1

Hayat, T., Khan, S.B., and Khan, M., 2007, "The Influence of Hall current on the Rotating Oscillating Flows of an Oldroyd-B Fluid in a Porous Medium," Nonlinear Dynamics, 47(4), 353-362. http://dx.doi.org/10.1007/s11071-006-9034-Z

Greek Symbols 
Hayat, T., Abbas, Z., and Sajid, M., 2006, "Series Solution for the Upper-Convected Maxwell Fluid over a Porous Stretching Plate," Physics Letters A, 358(5), 396-403.

http://dx.doi:10.1016/j.physleta.2006.04.117

Hayat, T., Ali, N., and Asghar, S., 2007, "Hall Effects on Peristaltic Flow of a Maxwell Fluid in a Porous Medium," Physics Letters A, 363(5), 397-403.

http://dx.doi:10.1016/j.physleta.2006.10.104

Hayat, T., and Qasim, M., 2010, "Influence of Thermal Radiation and Joule Heating on MHD Flow of a Maxwell Fluid in the Presence of Thermophoresis," International Journal of Heat and Mass Transfer, 53(21), 4780-4788.

http://dx.doi.org/10.1016/j.ijheatmasstransfer.2010.06.014

Kanth, A. R., and Aruna, K., 2008, "Differential Transform Method for Solving Linear and Non-linear Systems of Partial Differential Equations," Physics Letters A, 372(46), 6896-6898.

http://dx.doi:10.1016/j.physleta.2008.10.008

Mukhopadhyay, S., 2012, "Heat Transfer Analysis of the Unsteady Flow of a Maxwell Fluid over a Stretching Surface in the Presence of a Heat Source/Sink," Chinese Physics Letters, 29(5), 054703.

http://dx.doi.org/10.1088/0256-307X/29/5/054703

Mushtaq, A., Abbasbandy, S., Mustafa. M., Hayat, T., Alaedi A., 2016, "Numerical Solution for Sakiadis Flow of Upper-Convected Maxwell Fluid using Cattaneo-Christov Heat Flux Model," AIP Advances 6, 015208, 1-9.

http://dx.doi.org/10.1063/1.4940133

Odelu Ojjela., and Naresh Kumar, N., 2015, "Unsteady MHD Flow and Heat Transfer of Micropolar Fluid in a Porous Medium between Parallel Plates," Canadian Journal of Physics, 93(999), 880-887. http://dx.doi.org/10.1139/cjp-2013-0266

Patowary, G., and Sut, D. K.(2011) "Study the Effect of Variable Viscosity and Thermal Conductivity of Micropolar Fluid in a Porous Channel," Int. J. Comp. Tech. Appl, 2(5), 1247-1255.

Prasad, K.V., Vajravelu, K., and Datti, P.S., 2010, "The Effects of Variable Fluid Properties on the Hydro-magnetic Flow and Heat Ttransfer over a Non-linearly Stretching Sheet," International Journal of Thermal Sciences, 49(3), 603-610.

http://dx.doi.org/10.1016/j.ijthermalsci.2009.08.005

Rahaman, K., and Ramkissoon, H., 1995, "Unsteady Axial Viscoelastic Pipe Flows," Journal of Non-Newtonian Fluid Mechanics, 57(1), 2738.

\section{http://dx.doi.org/10.1016/0377-0257(94)01293-Q}

Rajagopal, K., and Srinivasa, A., 2000, "A Thermodynamic Frame Work for Rate type Fluid Models," Journal of Non-Newtonian Fluid Mechanics, 88(3), 207-227.

http://dx.doi.org/10.1016/S0377-0257(99)00023-3

Ramana Murthy, J. V., Srinivasacharyulu, N., and Odelu Ojjela, 2007, "Viscous Ffluid Flow between Two Parallel Plates with Periodic Suction and Injection," AMSE Journal Advances in Modelling Series B, 50(2), 29-37.

Rassoulinejad-Mousavi, S. M., Abbasbandy, S., 2011, "Analysis of Forced Convection in a Circular Tube Filled With a Darcy-BrinkmanForchheimer Porous Medium Using Spectral Homotopy Analysis Method," Journal of Fluids Engineering 133 (101207), 1-9.

\section{http://dx.doi.org/10.1115/1.4004998}

Rassoulinejad-Mousavi, S. M., Seyf H. R., and Abbasbandy, S., 2013, "Heat Transfer through a Porous Saturated Channel with Permeable Walls using Two-Equation Energy Model," Journal of Porous Media 16 (3), 241-254.

http://dx.doi.org/ 10.1615/JPorMedia.v16.i3.60

Rassoulinejad-Mousavi, S.M., Yaghoobi, H., 2014, "Effect of Nonlinear Drag Term on Viscous Dissipation in a Fluid Saturated Porous Medium Channel with Various Boundary Conditions at Walls," Arab J. Sci. Eng. 39, 1231-1240.

http://dx.doi.org/10.1007/s13369-013-0676-0

Rassoulinejad-Mousavi, S. M., Abbasbandy, S., Alsulam, H. H., 2014, "Analytical Flow Study of a Conducting Maxwell Fluid through a Porous Saturated Channel at Various Wall Boundary Conditions," Eur. Phys. J. Plus 133 (101207), 129:181. 1-10. http://dx.doi.org/10.1140/epjp/i2014-14181-4

Renardy, M., and Renardy, Y., 1986, "Linear Stability of Plane Couette Flow of an Upper Convected Maxwell Fluid,"Journal of non-newtonian fluid mechanics, 22(1), 23-33.

http://dx.doi.org/10.1016/0377-0257(86)80002-7

Renardy, M., 1997, "High Weissenberg Number Boundary Layers for the Upper Convected Maxwell Fluid," Journal of Non-Newtonian Fluid Mechanics, 68(1), 125-132.

http://dx.doi.org/10.1016/S0377-0257(96)01491-7

Sadeghy, K., Najafi, A.-H., and Saffaripour, M., 2005, "Sakiadis Flow of an Upper-Convected Maxwell Fluid," International Journal of NonLinear Mechanics, 40(9), 1220-1228.

http://dx.doi:10.1016/j.ijnonlinmec.2005.05.006

Seyf H. R., Rassoulinejad-Mousavi, S. M., 2011, “An Analytical Study for Fluid Flow in Porous Media Imbedded Inside a Channel with Moving or Stationary Walls Subjected to Injection/Suction," Journal of Fluids Engineering 133 (091203), 1-9 http://dx.doi.org/ 10.1115/1.4004822

Shateyi, S., Motsa, S., and Makukula, Z., 2015, "On Spectral Relaxation Method for Entropy Generation on a MHD Flow and Heat Transfer of a Maxwell Fluid.," Journal of Applied Fluid Mechanics, 8(1), 21-31.

Sutton, G.W. and Sherman, A., 1965. Engineering Magnetohydrodynamics. McGraw-Hill.

Terrill, R., and Shrestha, G., 1966, "Laminar Flow through a Channel with Uniformly Porous Walls of Different Permeability," Applied Scientific Research, 15(1), 440- 468.

http://dx.doi.org/10.1007/BF00411577

Umavathi, J. C., and Shekar, M. (2016). Combined Effect of Variable Viscosity and Thermal Conductivity on Free Convection Flow of a Viscous Fluid in a Vertical Channel using DTM. Meccanica, 51(1), 7186.

http://dx.doi.org/10.1007/s11012-015-0202-4

White Jr, F.M., Barfield, B. F., and Goglia, M. J., 1958, "Laminar Flow in a Uniformly Porous Channel," Journal of Applied Mechanics, 613617.

Zhou, J., 1986, "Differential Transformation and its Applications for Electrical Circuits," Huazhong University Press, Wuhan. 